\title{
The Acceptable Noise Level Benefit From Directionality for Listeners With Severe Hearing Loss
}

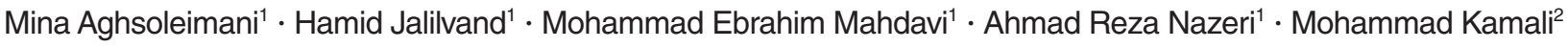 \\ ${ }^{\prime}$ Department of Audiology, School of Rehabilitation, Shahid Beheshti University of Medical Sciences, Tehran, \\ ${ }^{2}$ Department of Basic Sciences, School of Rehabilitation, Iran University of Medical Sciences, Tehran, Iran
}

Objectives. Directional microphone technology can enhance the speech intelligibility and listening comfort of listeners with hearing impairment. The main aim of this study is to investigate and compare the benefit derived by listeners with severe hearing loss from directional microphone technology with that derived by listeners with moderate hearing loss.

Methods. The acceptable noise levels (ANLs) of two groups of listeners with moderate or severe hearing impairment (17 subjects in each group) were measured under unaided, omnidirectional-baseline-aided, and directional-aided conditions.

Results. Although the absolute ANL of the listeners in the severe hearing loss group was significantly higher than that of the listeners in the moderate hearing loss group, their derived benefit was equivalent to that derived by the listeners in the moderate hearing loss group. ANL and hearing loss degree were significantly related. Specifically, the ANL increased with the severity of hearing loss.

Conclusion. Directional microphone technology can provide the benefits of listening comfort to listeners with severe hearing loss.

Keywords. Directional Hearing Aid; Hearing Aids; Acceptable Noise Level; Hearing Loss

\section{INTRODUCTION}

The human auditory system enables the detection and discrimination of sounds and provides speech intelligibility in noise, a necessary ability lacking in a listener with sensorineural hearing loss. Thus, the ability to discern speech from noise is the main demand of many listeners with sensorineural hearing loss. Directional microphone technology is the main digital signal processing (DSP) technology employed in modern digital hearing aids to improve speech intelligibility. Directional microphones utilize multiple microphones or ports (at least two microphones or ports) and spatially separate speech from noise to improve

- Received October 3, 2017

Revised January 8, 2018

Accepted January 11, 2018

- Corresponding author: Hamid Jalilvand

Department of Audiology, School of Rehabilitation, Shahid Beheshti

University of Medical Sciences, Damavand St.,Tehran, Iran

Tel: +98-21-7756-1721, Fax: +98-21-7759-1807

E-mail: hamidjalilvand@sbmu.ac.ir signal-to-noise ratio. By clarifying speech and lessening noise, this technology improves the listener's ability to understand speech in noisy environments. The ability of directional hearing aids to improve speech perception under noisy conditions has been demonstrated by different studies, which utilized different variables, tools, and methodologies [1]. These studies, however, investigated speech perception in noise among participants with normal hearing [2], and mild to moderate hearing loss [1]. By contrast, speech performance in participants with severe hearing loss has not been widely investigated.

Acceptable noise level (ANL) is a subjective measurement used to evaluate a subject's performance in noise. The ANL was introduced by Nabelek et al. [3] as a test for evaluating a subject's willingness to tolerate noise while listening to a running speech. It is quantified as the difference between the most comfortable level (MCL) of a running speech and the background noise level (BNL) of a competing babble noise tolerated by the subject $(\mathrm{ANL}=\mathrm{MCL}-\mathrm{BNL})$. Listeners with a lower value of ANL can tolerate a higher level of noise than listeners with a

Copyright $\odot 2018$ by Korean Society of Otorhinolaryngology-Head and Neck Surgery.

This is an open-access article distributed under the terms of the Creative Commons Attribution Non-Commercial License (http://creativecommons.org/licenses/by-nc/4.0)

which permits unrestricted non-commercial use, distribution, and reproduction in any medium, provided the original work is properly cited. 
higher value of ANL. A high ANL indicates that the listener cannot tolerate a high level of noise. If a special DSP algorithm of the user's hearing aid (for example, directional microphone) decreases noise, the user's ANL decreases relative to a baseline condition under which the algorithm is not used. The ANL benefit, which is obtained by subtracting the ANL obtained under a specific condition from the ANL obtained under the baseline condition, can be used to show the effectiveness of a special technology for reducing noise. In addition, it is indicative of a subject's listening comfort in noise. Thus, ANL can be used to evaluate the benefit provided by different hearing aid technologies. However, despite numerous studies on ANL and its application in various research designs, the belief that hearing loss and ANL result are unrelated continues to persist. In fact, all related studies on ANL have investigated subjects with normal hearing or mild to moderately severe sloping hearing loss. Studies on the ANL of subjects with severe hearing loss have not compared the ANL of the subjects under unaided and aided conditions [4] (for a thorough review, refer to [5]). A major original work from Nabelek et al. [6] investigated listeners with moderate sloping hearing loss. No study has compared the ANL results obtained under unaided or aided conditions of subjects with severe and less hearing loss. For example, a study on the ANL of participants with severe hearing loss did not present the details of hearing thresholds and did not compare the ANL results of the participants under unaided and aided conditions [7]. In addition, the real ear measurement (REM) was not used to verify amplification; thus, the conclusion and generality of the results of the study are unreliable. An Australian study [8], which included normal and hearing-impaired participants with different degrees of hearing losses, did not compare the ANL results obtained under unaided and aided conditions. Thus, the studies that utilized ANL to evaluate the effect of directional microphone technology have involved subjects with less than severe hearing loss.

On the other hand, there are some factors that can influence ANL, such as audiogram configuration [9], speech intelligibility [10], binaural versus monaural directional amplification [11], duration of hearing aid usage [11], personality type [12], and

\section{H I G H L I G H T S}

- Listeners with severe hearing loss still derived benefits from directional hearing aids that are equivalent to that derived by listeners with moderate hearing loss.

- The acceptable noise levels (ANLs) of the severe hearing loss group are significantly different under unaided and omnidirectional-baseline conditions.

- Our results consistently showed that ANL is related to the degree of hearing loss; severe hearing loss is associated with high ANL. meaning of competing speech noise for the BNL measurement [11]. The slope of the audiogram affect the ANL outcome [9]. Listeners with good low frequency hearing thresholds had high ANLs. ANL measures increase with the increasing difference between the averages of hearing thresholds for high and low frequencies. This result suggested that various mechanisms likely underlie the ANL outcomes of listeners with different audiogram slopes or hearing losses. In addition, openness personality dimension [12], binaural directional amplification, less meaningful competing noise, and long experience of hearing aid usage [11] allow users to accept a greater amount of background noise.

Therefore, we designed the present study to address the inadequacies of the above studies on ANL: (1) few studies have investigated the benefits derived by listeners with severe hearing loss from directional hearing aids; (2) no study has compared the ANL values of subjects with different degrees of hearing loss (mild-moderate vs. severe) under unaided and aided conditions; (3) no study has utilized ANL to investigate the benefit derived from directional hearing aids by subjects with severe hearing loss.

Finally, the main questions of the present study are as follows. (1) Are the ANLs of subjects with mild-moderate and severe hearing loss under unaided and aided conditions significantly different? (2) Are the ANL benefits derived by subjects with moderate and severe hearing from the use of directional microphone technology significantly different? In summary, our study aims to compare the performance of two groups of subjects with moderate or severe hearing loss on the basis of ANL results under unaided, omnidirectional-aided, and directional-aided conditions.

\section{MATERIALS AND METHODS}

\section{Participants}

A total of 34 participants with hearing loss (19 males and 15 females) and who used hearing aids (with at least 2-year experience of hearing aid amplification) participated in the present study. All participants, who had symmetric sensorineural hearing loss (less than $10 \mathrm{~dB}$ difference between ears across 250$8,000 \mathrm{~Hz}$ ), were unilateral hearing aid user. The word recognition scores of all subjects were higher than $72 \%$ (in terms of speech audiometry results from the 50 -word list) and all their aided thresholds across hearing frequencies were in range of 20-40 dB (HL) (Fig. 1). All measurements were taken unilaterally (19 right and 15 left ears). The first group (G1) included 17 subjects with moderate sensorineural hearing loss (Fig. 1) and the second group (G2) included 17 subjects with severe sensorineural hearing loss (Fig. 1). The hearing loss range of the participants in G1 ranged from mild to moderate in low frequencies that extended to severe hearing loss in high frequencies. The 

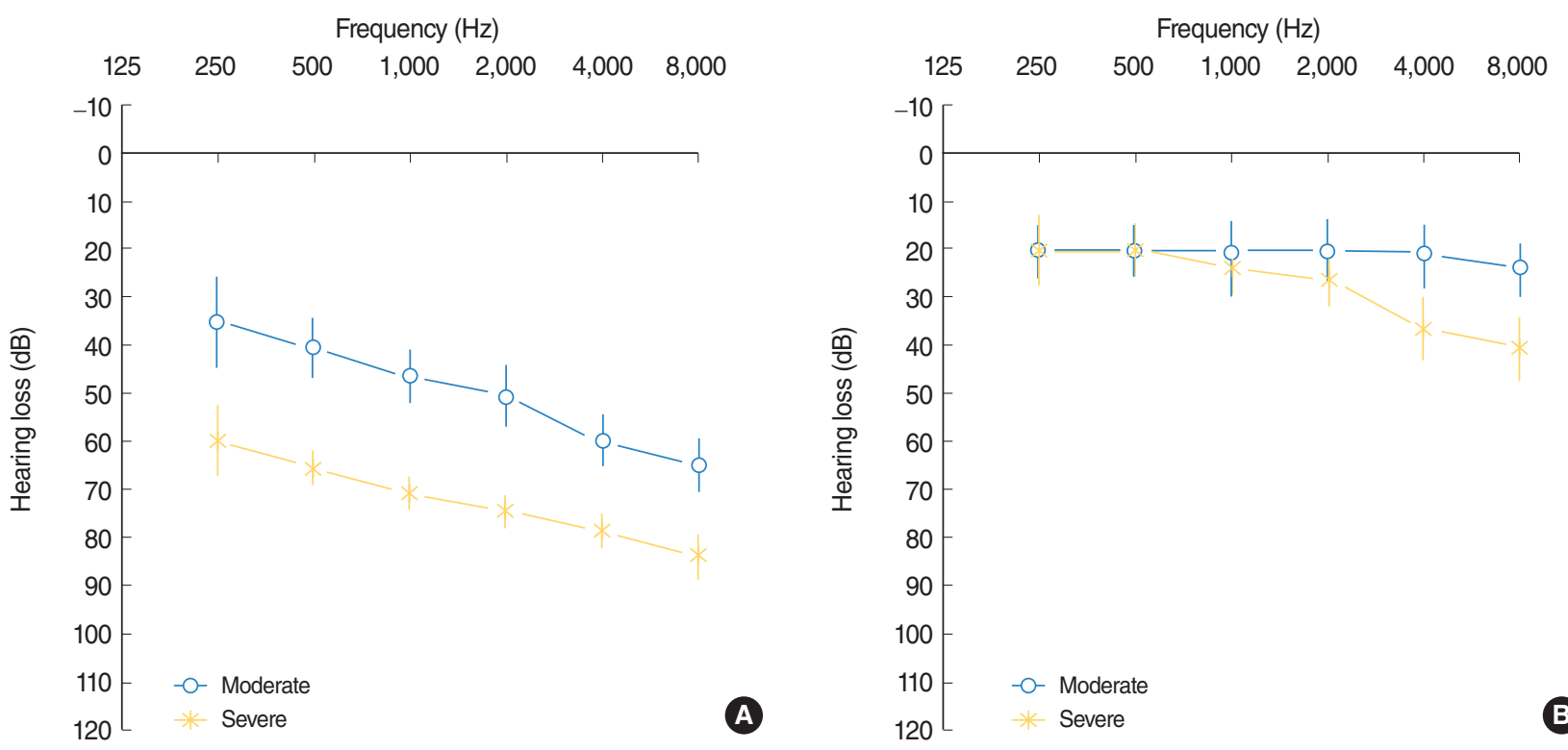

Fig. 1. The mean audiograms (A) and the aided audiograms (B) of moderate (G1) and severe (G2) hearing loss. Bars show the standard deviations.

hearing loss range of the participants in G2 ranged from moderate to severe in low frequencies and severe to profound in high frequencies. The mean age of the participants in G1 was $63 \pm 5$ years and that of participants in G2 was $66 \pm 5$ years. Age was not statistically and significantly different between groups.

\section{Hearing aid fitting}

The study was accomplished in two phases: (1) phase 1 included the invitation and interview for study participation. To control for cognitive effects, each participant was evaluated using the Mini-Mental State Examination (MMSE). The criterion in the present study was a cutoff score of 23 or higher on the MMSE [13]. Basic audiometry evaluation was accomplished after obtaining signed written consent from the participants. Participants were subjected to otoscopy evaluation, immittance audiometry, pure tone audiometry, and speech audiometry. An ear impression was taken from each participant for the fabrication of an earmold. This session lasted 1.5-2 hours on average for every participant. (2) Phase 2 included hearing aid fitting, REM, and ANL measurements. Motion 301 P BTE, a Siemens BTE hearing aid (Siemens, Munich, Germany) was used as the hearing aid in this study. FP35 hearing aid analyzer (Frye Electronics, Beaverton, OR, USA) was used for REM. Hearing aid amplification was adjusted by National Acoustic Laboratories-Nonlinear 2 (NAL-NL2) targets for inputs of 50, 65, and $80 \mathrm{~dB}$ sound pressure level (SPL). The stimulus Digital Speech (Frye Electronics Co. Beaverton, OR, USA) was used for REM measurements. All amplification measures where in the range of $\pm 3 \mathrm{~dB}$ from NAL-NL2 targets for the mentioned inputs in G1 participants. However, in G2 participants, the NAL-NL2 targets were not provided in the range of $\pm 3 \mathrm{~dB}$ for high frequency ranges
$(4,000-8,000 \mathrm{~Hz})$. In this group, hearing aid was fitted in maximum gain for high frequency range. Nevertheless, on average, the hearing aid amplifications for all participants of G2 were 12, 10 , and $7 \mathrm{~dB}$ less than NAL-NL2 targets for inputs of 50, 65, and $80 \mathrm{~dB}$ SPL in high frequency range $(4,000-8,000 \mathrm{~Hz})$. In addition, the maximum pressure output was set in accordance with the NAL-NL2 targets for warble pure tone at an input 90 $\mathrm{dB}$ SPL. Given the effect(s) of vent or open fitting on the performance of the directional microphone [14-16], an unvented earmold was used for all participants. ANL measurements were randomly performed under three various conditions: unaided, omnidirectional-baseline, and directional conditions. This session lasted 1.5-2 hours on average with a rest period during ANL testing if necessary. During all measurements, the untested ear was occluded by the participant's own ear impression. The ethical committee of the Shahid Beheshti University of Medical Sciences (IR.SBMU.RETECH.REC.1396.182) approved all the used procedures, methods, and tests.

\section{Test materials}

\section{Acceptable noise level}

ANL was introduced by Nabelek et al. [3], and is a quantitative measurement of a listener's noise tolerance. ANL is composed of two measurements: MCL and BNL. For MCL measurement, a running story was presented to the subject through a front speaker $1 \mathrm{~m}$ away and starting at $30 \mathrm{~dB}$ HL. Then, a clinical technician asked the subject to adjust the level of speech to their comfort preference by showing his/her thumb up or down to increase or decrease the level of speech. Speech was adjusted in decrements or increments of $5 \mathrm{~dB}$. Before testing, a written document was given to the subject to clarify the various stages of 
test and the subject's task during testing. In addition, the task was explained orally before MCL measurement. At the range of the preferred comfort level, the decrement or increment changed to $2 \mathrm{~dB}$ steps. After determining the preferred MCL of the participant, the level of the running story was increased and decreased significantly (i.e., $10 \mathrm{~dB}$ ) to show the upper and lower limits of the sound to the subject. The subject was asked again to adjust the sound level to their preference for MCL. MCL measurement was performed thrice and the average MCL was recorded as the subject's MCL measure. For BNL measurement, the running story was presented to the subject at the obtained MCL and a 12-talker babble noise was added and played at 30 dB HL. During this stage, the subject was asked to adjust the level of noise to a level that they could not tolerate and could not follow the speech higher than that level. After the initial determination of BNL, the level steps were decreased from 5 to 2 $\mathrm{dB}$. The BNL measurement was obtained thrice, and the average was calculated and recorded as the final BNL. Finally, to obtain ANL, BNL measurement was subtracted from MCL measurement: $\mathrm{ANL}=\mathrm{MCL}-\mathrm{BNL}$.

To determine the effect of amplification, the ANL benefit was calculated for each group by subtracting the ANL obtained under the omnidirectional-baseline condition from the unaided condition. Similarly, the ANL measure obtained under the directional condition was subtracted from that obtained under the omnidirectional-baseline condition to calculate the ANL benefit under the directional condition. The Persian version of ANL, which was developed and validated by Ahmadi et al. [17], was used in the present study.

\section{Statistical method}

IBM SPSS ver. 24.0 (IBM Corp., Armonk, NY, USA) was used to analyze the study data. Descriptive statistical parameters, such as the mean and standard deviation of the ANL results were considered. A mixed repeated-measurement analysis of variance test was performed to determine statistically significant differences at the 0.05 level in ANL under different conditions. In addition, various conditions were compared using the Bon- ferroni test as a post-hoc analysis. In this analysis, the withinsubject factor is six various conditions ( 2 groups $\times 3$ conditions). The paired samples $t$-test was used to compare ANL benefits. In addition, the Pearson test was performed to evaluate the probable correlation between results.

\section{RESULTS}

\section{Most comfortable level}

Table 1 shows the MCL ranges and means (and standard deviations [SDs]) of G1 and G2 groups under the unaided, omnidirectional-baseline-aided, and directional conditions. As shown in

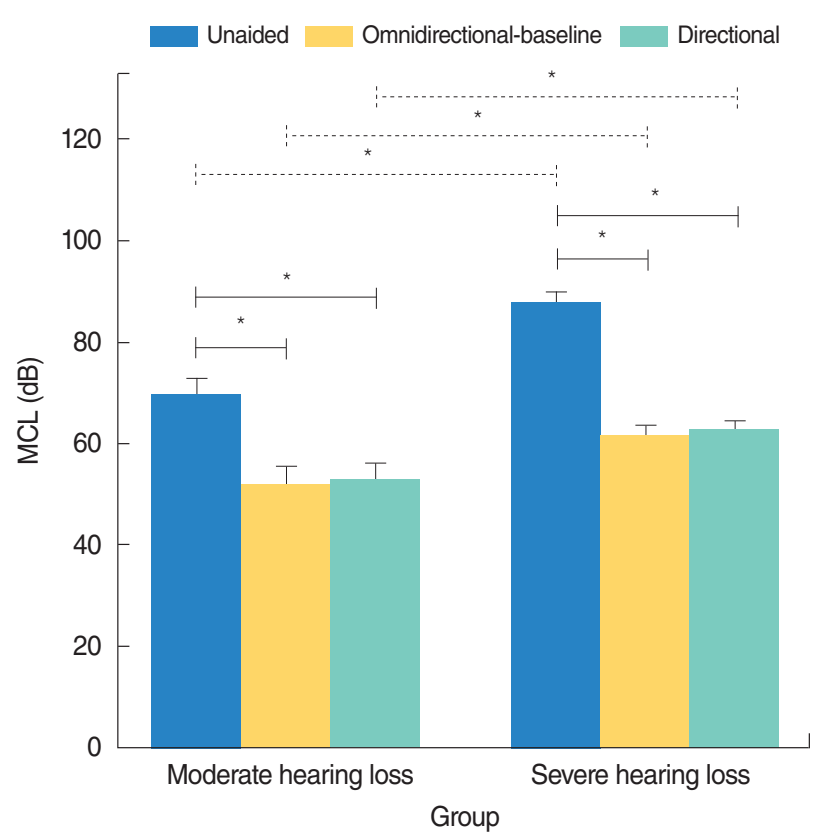

Fig. 2. Mean most comfortable level (MCL) values of moderate hearing loss (G1) and severe hearing loss (G2) under unaided, omnidirectional-baseline, and directional conditions. Error bars indicate $95 \%$ confidence intervals. ${ }^{*} P<0.001$, significant differences between intra- and intergroup results, respectively.

Table 1. The range and mean of MCL, BNL, and ANL for two groups of hearing loss (G1 and G2) under unaided, omnidirectional-aided, and directional-aided conditions

\begin{tabular}{|c|c|c|c|c|c|c|}
\hline \multirow{2}{*}{ Variable } & \multicolumn{3}{|c|}{ G1 } & \multicolumn{3}{|c|}{ G2 } \\
\hline & $\begin{array}{l}\text { Unaided } \\
\text { condition }\end{array}$ & $\begin{array}{l}\text { Omni-baseline } \\
\text { condition }\end{array}$ & $\begin{array}{l}\text { Directional } \\
\text { condition }\end{array}$ & $\begin{array}{l}\text { Unaided } \\
\text { condition }\end{array}$ & $\begin{array}{c}\text { Omni-baseline } \\
\text { condition }\end{array}$ & $\begin{array}{l}\text { Diretional } \\
\text { condition }\end{array}$ \\
\hline $\mathrm{MCL}(\mathrm{dB} H \mathrm{H})$ & $\begin{array}{c}56 \text { to } 79 \\
(69.76 \pm 5.76)\end{array}$ & $\begin{array}{c}29 \text { to } 62 \\
(52.00 \pm 7.13)\end{array}$ & $\begin{array}{c}30 \text { to } 61 \\
(52.65 \pm 6.80)\end{array}$ & $\begin{array}{c}80 \text { to } 94 \\
(88.00 \pm 3.70)\end{array}$ & $\begin{array}{c}54 \text { to } 68 \\
(61.76 \pm 3.61)\end{array}$ & $\begin{array}{c}54 \text { to } 69 \\
(62.65 \pm 3.55)\end{array}$ \\
\hline $\mathrm{BNL}(\mathrm{dB} H \mathrm{HL})$ & $\begin{array}{c}58 \text { to } 80 \\
(69.05 \pm 6.09)\end{array}$ & $\begin{array}{c}31 \text { to } 63 \\
(51.70 \pm 7.61)\end{array}$ & $\begin{array}{c}35 \text { to } 65 \\
(55.17 \pm 7.01)\end{array}$ & $\begin{array}{c}74 \text { to } 89 \\
(83.82 \pm 4.05)\end{array}$ & $\begin{array}{c}52 \text { to } 66 \\
(58.94 \pm 3.73)\end{array}$ & $\begin{array}{c}56 \text { to } 68 \\
(63.05 \pm 3.07)\end{array}$ \\
\hline $\mathrm{ANL}(\mathrm{dB})$ & $\begin{array}{c}-2 \text { to } 6 \\
(0.82 \pm 2.48)\end{array}$ & $\begin{array}{c}-3 \text { to } 5 \\
(0.29 \pm 2.61)\end{array}$ & $\begin{array}{c}-5 \text { to } 1 \\
(-2.53 \pm 2.15)\end{array}$ & $\begin{array}{c}1 \text { to } 8 \\
(4.18 \pm 2.35)\end{array}$ & $\begin{array}{c}-1 \text { to } 9 \\
(2.88 \pm 2.44)\end{array}$ & $\begin{array}{c}-4 \text { to } 9 \\
(-0.41 \pm 1.77)\end{array}$ \\
\hline
\end{tabular}

Values are presented as range (mean \pm standard deviation).

MCL, most comfortable level; BNL, background noise level; ANL, acceptable noise level; G1, moderate hearing loss; G2, severe hearing loss. 
Fig. 2, the MCL means of the moderate hearing loss group are significantly lower than that of the severe hearing loss group under all three mentioned conditions $\left(\mathrm{F}_{1.1,36}=867.42, P<0.001\right.$, $\left.\eta^{2}=0.96\right)$. Overall, significant differences exist between the unaided and omnidirectional-baseline conditions $(P<0.001)$, the unaided and directional conditions $(P<0.001)$, and the omnidirectional-baseline and directional conditions $(P<0.001)$. Although the maximum difference between the means of the two groups are $18.24 \mathrm{~dB}$ under the unaided condition, the difference between means of MCLs under both omnidirectional-baseline and directional conditions is nearly same $(9.76$ and $10 \mathrm{~dB}$, respectively).

The MCL measurements of the moderate group are significantly different under unaided and omnidirectional-baseline conditions $(P<0.001)$ and unaided and directional conditions $(P<0.001)$. There is not a significant difference between omnidirectional-baseline and directional conditions $(P=0.330)$ (Fig. 2). Similarly, significant differences exist in the MCL measurements of the severe hearing loss group between the unaided and omnidirectional-baseline conditions $(P<0.001)$, the unaided and directional conditions $(P<0.001)$. Again, the MCLs of omnidirectional-baseline-aided and directional-aided conditions are not statistically significant $(P=0.330)$ (Fig. 2).

\section{Absolute ANL}

Under the unaided, omnidirectional-baseline-aided, and directional conditions, the ANL ranges of the moderate and severe hearing loss groups are -2 to 6 vs. 1 to $8 ;-3$ to 5 vs. -1 to 9 ; and -5 to 1 vs. -4 to $9 \mathrm{~dB}$, respectively (Table 1 ). As indicated in Table 1, the ANL means (and SDs) of two different groups are $0.82 \pm 2.48$ vs. $4.18 \pm 2.35 ; 0.29 \pm 2.61$ vs. $2.88 \pm 2.44$; and $-2.53 \pm 2.15$ vs. $-0.41 \pm 1.77 \mathrm{~dB}$ under the unaided, omnidirectional-baseline, and directional conditions, respectively. Comparison of the ANL means shows that the ANL means of the G2 group are significantly higher than that of the G1 hearing loss group under all three mentioned conditions $\left(\mathrm{F}_{1,32}=14.29, P=\right.$ $0.001, \eta^{2}=0.30$ ) (Fig. 3). There are significant differences between the unaided and omnidirectional-baseline conditions $(P=0.039)$, the unaided and directional conditions $(P<0.001)$, and the omnidirectional-baseline and directional conditions $(P<0.001)$. The minimum and maximum differences between the means of the two groups are 2.12 and $3.36 \mathrm{~dB}$, which are belong to the unaided and directional conditions, respectively.

As illustrated in Fig. 3, the ANL measurements of the moderate group are not significantly different under unaided and omnidirectional-baseline conditions $(P=0.110)$ but are significantly different under unaided and directional conditions $(P<0.001)$ and omnidirectional-baseline and directional conditions $(P<$ 0.001). In G2 group, significant differences are observed in the ANL measurements of the severe hearing loss group between the unaided and omnidirectional-baseline conditions $(P=0.023)$, the unaided and directional conditions $(P<0.001)$, and the om-

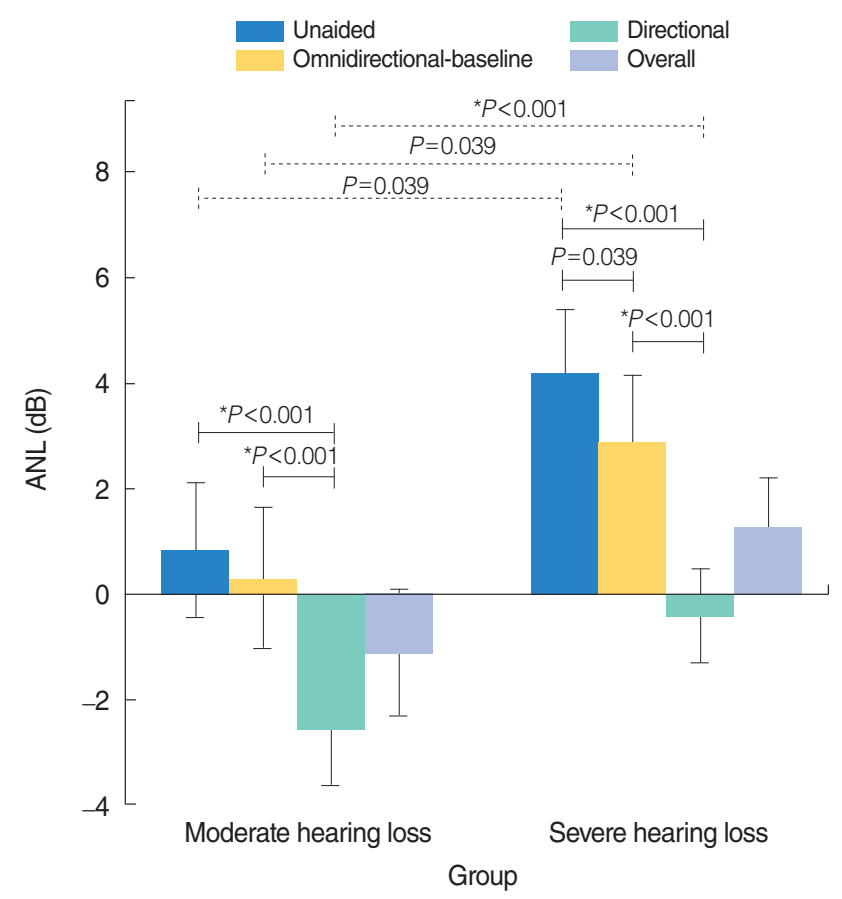

Fig. 3. Mean acceptable noise level (ANL) values of moderate hearing loss (G1) and severe hearing loss (G2) under unaided, omnidirectional-baseline, and directional conditions. The overall condition is average of ANLs under omnidirectional-baseline and directional conditions. Error bars indicate 95\% confidence intervals. *Significant differences between intra- and intergroup results, respectively.

nidirectional-baseline-aided and directional-aided conditions $(P<0.001)$ (Fig. 3).

\section{ANL correlation}

The pooled ANL results revealed significant positive correlations between unaided ANL and omnidirectional-aided ANL $(r=0.75$, $P<0.001)$, between unaided ANL and directional-aided ANL $(r=0.76, P<0.001)$, and between omnidirectional ANL and directional-aided ANL $(r=0.83, P<0.001)$. Specifically, for each group (G1 vs. G2), a significant positive correlation exists between unaided ANL and omnidirectional ANL $(r=0.63, P=$ 0.003 vs. $r=0.66, P=0.003$ ), between unaided ANL and directional-aided ANL ( $r=0.63, P=0.006$ vs. $r=0.73, P=0.001)$, and between omnidirectional-aided ANL and directional-aided ANL ( $r=0.90, P<0.001$ vs. $r=0.62, P=0.008)$. Pearson correlation demonstrated a significant positive correlation between the hearing thresholds of all frequencies, the average of hearing thresholds of 250 and $500 \mathrm{~Hz}$, and the average of hearing thresholds for frequencies 1,000, 2,000, 4,000, and 8,000 Hz and ANL under the three study conditions (Table 2).

\section{ANL benefit}

The results showed that the ANL benefit under omnidirectionalbaseline and directional conditions in the moderate hearing loss group is $-0.52 \pm 2.06 \mathrm{~dB}$ and $-3.35 \pm 1.99 \mathrm{~dB}$, respectively, and 
Table 2. Pearson correlation between hearing thresholds (in each frequency), and average of hearing thresholds in low and high frequencies $(250,500,1,000$ through $8,000 \mathrm{~Hz})$ and ANL under unaided, omnidirectional-aided, and directional-aided conditions

\begin{tabular}{|c|c|c|c|c|c|c|}
\hline \multirow{2}{*}{ Frequency $(\mathrm{Hz})$} & \multicolumn{6}{|c|}{ ANL } \\
\hline & Unaided condition & $P$-value & Omnidirectional condition & $P$-value & Directional condition & $P$-value \\
\hline 250 & 0.44 & 0.009 & 0.37 & 0.027 & 0.44 & 0.008 \\
\hline 500 & 0.51 & 0.002 & 0.46 & 0.006 & 0.48 & 0.004 \\
\hline 1,000 & 0.53 & 0.001 & 0.49 & 0.001 & 0.52 & 0.001 \\
\hline 2,000 & 0.50 & 0.002 & 0.45 & 0.006 & 0.50 & 0.002 \\
\hline 4,000 & 0.48 & 0.004 & 0.42 & 0.012 & 0.46 & 0.006 \\
\hline 8,000 & 0.40 & 0.016 & 0.42 & 0.012 & 0.38 & 0.024 \\
\hline $\begin{array}{l}\text { Average of hearing thresholds } \\
\text { of } 250 \text { and } 500\end{array}$ & 0.49 & 0.003 & 0.43 & 0.011 & 0.47 & 0.004 \\
\hline $\begin{array}{l}\text { Average of frequencies } 1,000 \\
\text { through } 8,000\end{array}$ & 0.51 & 0.002 & 0.47 & 0.004 & 0.49 & 0.003 \\
\hline
\end{tabular}

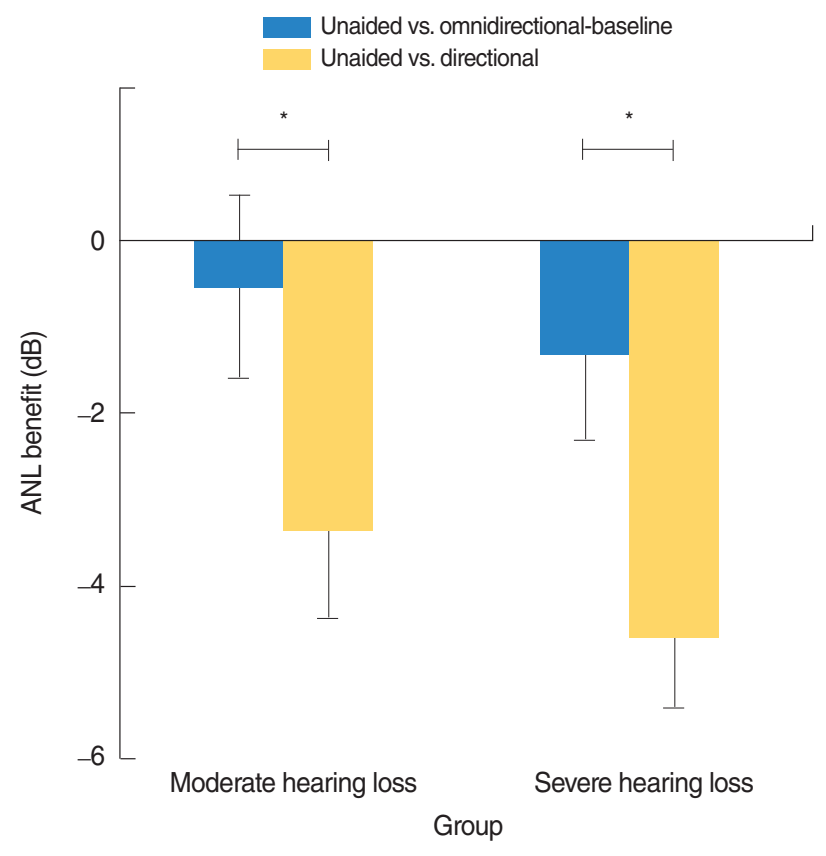

Fig. 4. Mean acceptable noise level (ANL) benefit of each group under omnidirectional and directional conditions. ANL benefit derived under omnidirectional and directional conditions are shown as blue and yellow columns, respectively. ${ }^{\star} P<0.001$, significant differences between results.

that for the severe hearing loss group is $-1.29 \pm 1.96 \mathrm{~dB}$ and $-4.58 \pm 1.58 \mathrm{~dB}$, respectively (Fig. 4). ANL benefit is significantly different between the omnidirectional and directional conditions for each group ( $P<0.001$ for both groups) (Fig. 4). For each group, the ANL benefit derived under directional condition is higher than that derived under omnidirectional condition (Fig. 4). However, the ANL benefits of the two groups are not significantly different $(P=0.270$ and $P=0.060$ for the omnidirectional and directional ANL benefits, respectively).

\section{DISCUSSION}

This study demonstrated that listeners with severe hearing loss still derived benefits from directional hearing aids that are equivalent to that derived by listeners with moderate hearing loss. Although their unaided and aided ANLs were significantly higher than that of subjects with moderate hearing loss, the participants with severe hearing loss obtained almost equal benefit from directional hearing aid. While unchanging their MCLs, directional microphone technology changes just their BNLs and consequently their ANLs. This result suggested that a directional hearing aid, by reducing noise through spatial separation, could provide listening comfort to listeners with severe hearing loss in a noisy environment. A similar study with different study design [1] utilized the connected speech test to investigate the potential benefit of directional hearing aids for subjects with severe hearing loss. The study found that directional hearing aids provided significant benefits for hearing-impaired people in a complex listening environment. The directional hearing aid can provide listening comfort without degrading speech intelligibility. However, directionality benefits obtained under laboratory and reallife conditions are inconsistent. Additional research under reallife conditions is necessary to determine the practical benefits of directional hearing aids for listeners with severe hearing loss.

Interestingly, Nabelek et al. [6] reported that unaided or aided listening conditions do not provide significantly different ANL results, a finding that is duplicated in the present study. Although we obtained a similar result for the moderate hearing loss group, the ANLs of the severe hearing loss group are significantly different under unaided and omnidirectional-baseline conditions. This inconsistent finding might be explained by multi-factors such as the different characteristics of hearing loss degrees, usage and benefit from speech information, and type of speech amplification and presentation. As hearing loss increases, the audibility, dynamic range, frequency resolution, and temporal resolution are decrease. In addition, using speech information in each frequency region decreases when degree of hearing loss in- 
creases [18]. More importantly, the fine characteristics and frequency response of speech in noise, which is amplified by a hearing aid (based on NAL-NL2 rationale) [19], are totally different from the same speech that presented from an audiometer [20-22]. The participants in the study by Nabelek et al [6]. had mild to moderate sloping hearing loss, whereas those in our study had moderate to severe hearing loss. ANL is consistently related to the audiogram configuration [9]. In addition, our results consistently showed that ANL is related to the degree of hearing loss: severe hearing loss is associated with high ANL. This finding is consistent with those of other studies $[8,9,23]$ and demonstrates the robable relationship between Pure Tone Average (PTA) and ANL: subjects with poor PTA have high ANLs. In all these studies, subjects with mild to severe sloping hearing loss were evaluated, whereas our study considered two groups with distinctly different degrees of hearing loss. Consistent with the results of previous studies, our results suggested that poor PTA thresholds are associated with high ANLs. Our finding on the positive correlation between ANL and the hearing thresholds of subjects is completely consistent with that of a recent study that evaluated the relationship between ANL and hearing thresholds [9] among people with mild or moderate sloping hearing losses. These findings suggested that, in addition to hearing aid technology, the degree of hearing loss could affect ANL. Thus, the degree of hearing loss should be considered when aided ANL is estimated on the basis of unaided ANL results.

Finally, to control the influencing factors on ANL (as mentioned in Introduction), the ANL measurements were accomplished in the study groups while controlling the factors. We measured ANL for monaural amplification with using 12-talker noise as the competing noise among participants with at least 2-year experience of hearing aid amplification. Unfortunately, the personality type could not be controlled because there is not the valid Persian questionnaire. Therefore, the obtained results are limited to the study condition. Further research is needed to investigate other possible conditions. Also, the above results were obtained under a specific listening condition without any reverberation and with a hearing aid model with its own directional microphone technology. Thus, the results of the present study could not be generalized to all hearing-impaired subjects under various amplification conditions. The hearing aid DSP can affect speech intelligibility under the condition that speech and noise both originate from the listener's front [24].

The results of the present study showed that, first, ANL and pure tone thresholds are related. Second, although the absolute ANL of the listeners in the severe hearing loss group was significantly higher than that of the listeners in the moderate hearing loss group, their derived benefit was equivalent to that derived by the listeners in the moderate hearing loss group.

\section{CONFLICT OF INTEREST}

No potential conflict of interest relevant to this article was reported.

\section{REFERENCES}

1. RickettsTA, Hornsby BW. Directional hearing aid benefit in listeners with severe hearing loss. Int J Audiol. 2006 Mar;45(3):190-7.

2. Leeuw AR, Dreschler WA. Advantages of directional hearing aid microphones related to room acoustics. Audiology. 1991;30(6):330-44.

3. Nabelek AK, Tucker FM, Letowski TR. Toleration of background noises: relationship with patterns of hearing aid use by elderly persons. J Speech Hear Res. 1991 Jun;34(3):679-85.

4. Jalilvand H, Pourbakht A, Jalaee S. The relationship between hearing aid frequency response and acceptable noise level in patients with sensorineural hearing loss. Adv Biomed Res. 2015 Nov;4:256.

5. Olsen SO, Brannstrom KJ. Does the acceptable noise level (ANL) predict hearing-aid use? Int J Audiol. 2014 Jan;53(1):2-20.

6. Nabelek AK, Freyaldenhoven MC, Tampas JW, Burchfiel SB, Muenchen RA. Acceptable noise level as a predictor of hearing aid use. J Am Acad Audiol. 2006 Oct;17(9):626-39.

7. Ho HC, Wu YH, Hsiao SH, Zhang X. Acceptable noise level (ANL) and real-world hearing-aid success in Taiwanese listeners. Int J Audiol. 2013 Nov;52(11):762-70.

8. Walravens E, Keidser G, Hartley D, Hickson L. An Australian version of the acceptable noise level test and its predictive value for successful hearing aid use in an older population. Int J Audiol. 2014 Feb;53 Suppl 1:S52-9.

9. Jonas Brannstrom K, Olsen SO. The acceptable noise level and the pure-tone audiogram. Am J Audiol. 2017 Mar;26(1):80-7.

10. Recker KL, Micheyl C. Speech intelligibility as a cue for acceptable noise levels. Ear Hear. 2017 Jul-Aug;38(4):465-74.

11. Kim JH, Lee JH, Lee HK. Advantages of binaural amplification to acceptable noise level of directional hearing aid users. Clin Exp Otorhinolaryngol. 2014 Jun;7(2):94-101.

12. Franklin C, Johnson LV, White L, Franklin C, Smith-Olinde L. The relationship between personality type and acceptable noise levels: a pilot study. ISRN Otolaryngol. 2013 Nov;2013:902532.

13. Ansari NN, Naghdi S, Hasson S, Valizadeh L, Jalaie S. Validation of a mini-mental state examination (MMSE) for the Persian population: a pilot study. Appl Neuropsychol. $2010 \mathrm{Jul} ; 17(3): 190-5$.

14. Kuk F, Keenan D. How do vents affect hearing aid performance? Hear Rev. 2006 Feb;13(2):34-42.

15. Magnusson L, Claesson A, Persson M, Tengstrand T. Speech recognition in noise using bilateral open-fit hearing aids: the limited benefit of directional microphones and noise reduction. Int J Audiol. 2013 Jan;52(1):29-36.

16. Winkler A, Latzel M, Holube I. Open versus closed hearing-aid fittings: a literature review of both fitting approaches. Trends Hear. 2016 Feb;20. https://doi.org/10.1177/2331216516631741.

17. Ahmadi A, Fatahi J, Keshani A, Jalilvand H, Modarresi Y, Jalaie S. Developing and evaluating the reliability of acceptable noise level test in Persian language. Sci J Rehabil Med. 2015;4(2):109-17.

18. Hornsby BW, Johnson EE, Picou E. Effects of degree and configuration of hearing loss on the contribution of high- and low-frequency speech information to bilateral speech understanding. Ear Hear. 2011 Sep-Oct;32(5):543-55.

19. Keidser G, Dillon H, Flax M, Ching T, Brewer S. The NAL-NL2 prescription procedure. Audiol Res. 2011 Mar;1(1):e24. 
20. McRackan TR, Ahlstrom JB, Clinkscales WB, Meyer TA, Dubno JR Clinical implications of word recognition differences in earphone and aided conditions. Otol Neurotol. 2016 Dec;37(10):1475-81.

21. Christensen L, Lee L, Humes L. Can clinical word-recognition measures predict aided word recognition? Am Audit Soc Bull. 1994; 19(1):11, 16.

22. Dillon H, Byrne D, Upfold L. The reliability of speech discrimination testing in relation to hearing aid candidacy. J Otolaryngol Soc Aust.
1982;5(2):81-4

23. Olsen SO, Lantz J, Nielsen LH, Brannstrom KJ. Acceptable noise level (ANL) with Danish and non-semantic speech materials in adult hearing-aid users. Int J Audiol. 2012 Sep;51(9):678-88.

24. Peeters H, Kuk F, Lau CC, Keenan D. Subjective and objective evaluation of noise management algorithms. J Am Acad Audiol. 2009 Feb;20(2):89-98. 